\title{
Who is eating whom? Morphology and feeding type determine the size relation between planktonic predators and their ideal prey
}

\author{
Kai W. Wirtz* \\ Institute of Coastal Research, Helmholtz-Zentrum Geesthacht, Germany
}

\begin{abstract}
Body size determines the position of organisms in plankton food webs. The mass or diameter ratio between predators and their optimal prey is therefore a central element of size-based models, which attempt to link consumer groups across trophic levels. Despite a renaissance of size-based approaches in plankton ecology, however, this relation still lacks a generic and also mechanistically sound formulation. An empirically derived constant value of this ratio cannot describe the wide scatter in optimal prey diameter for specific predator size classes, especially in the mesozooplankton range. In this study, I propose that a given morphometric ratio between feedingrelated apparatus and total body volume decreases when predator size increases. This ratio decrease is due to the additional need for structural components in larger organisms for maintaining intra-body transport. Non-isometric scaling results in a non-linear dependency of optimal prey size on predator diameter. This dependency defines an average relation which enables the quantitative definition of feeding mode. This new trait variable explains a component of the variability in optimal prey diameter that is independent of predator diameter. Feeding mode as a trait can be interpreted as activity during grazing, mostly in terms of speed regulation in swimming or in feeding-current generation. Feeding mode, in concert with the classical trait predator size, accurately determines optimal prey size. This was extensively tested using literature data for the entire plankton domain. The theory predicts increasing feeding activity in larger consumer species. It elucidates how successional shifts in the composition of zooplankton communities are linked to weakly coupled changes in mean body size and feeding mode.
\end{abstract}

KEY WORDS: Zooplankton grazing - Optimal prey size $\cdot$ Biomechanics $\cdot$ Size-based model

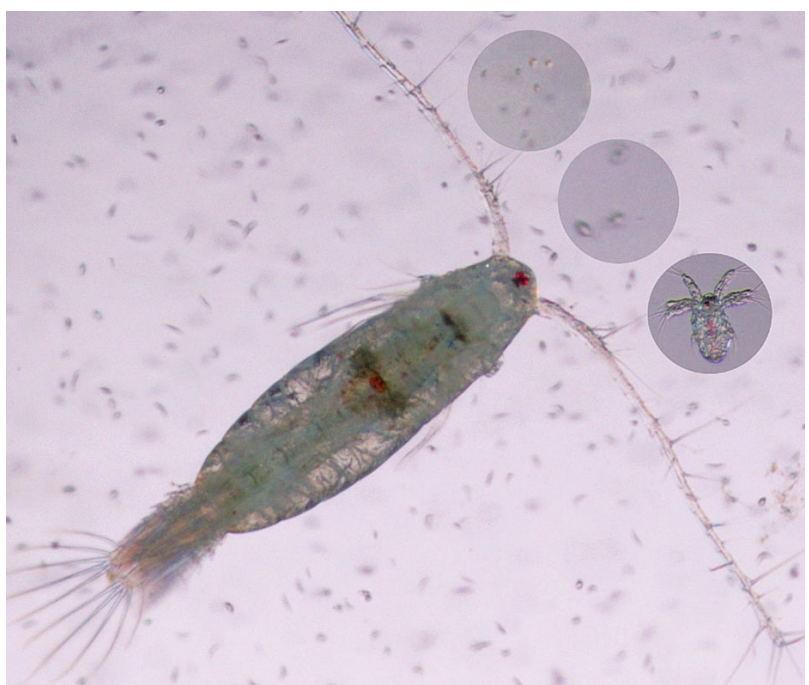

Morphological constraints largely determine which prey can be ingested by planktonic predators such as Acartia tonsa (shown here with Rhodomonas sp., Oxyrrhis sp. and a nauplius).

Photo: R. Schüller

\section{INTRODUCTION}

The structure of aquatic food webs is intimately connected to the body size of their constituent organisms (Brooks \& Dodson 1965, Woodward et al. 2005). To a considerable degree, size defines the trophic level of consumers and their prey. Prey size spectra of the dominant predator species in a system may reveal overlapping and empty niches. This translates into changing energy flows between trophic levels, potential mortality of prey species, failure in predator recruitment, or the success of an invader within a residing predator community. Body size is thus a fundamental ecological trait and defines a key compo- 
nent in our approaches to understanding food web structure and dynamics with models.

Aquatic ecosystem models increasingly address size as a trait, a strategy that has already been settled for non-planktonic food webs (Cohen et al. 1993, Woodward \& Hildrew 2002, Andersen \& Beyer 2006). In plankton ecology, models in general focus on autotrophic and nutrient-related processes. As a consequence, they hardly address changes in the matching between consumer preferences and prey availability, nor feeding interactions across more than 2 trophic levels. Size-oriented model studies that could address such issues were the exception for a long time. After the pioneer works of Moloney \& Field (1991) and Armstrong (2003), a cohort of size-based plankton food web models has recently been developed (e.g. Baird 2010, Fuchs \& Franks 2010, Zhou et al. 2010, Banas 2011). Apart from methodological differences, these spectral or multi-species size-based models all acknowledge the role of diversity in plankton ecosystems (Bruggeman \& Kooijman 2007). When describing size diversity within trophic levels, however, these models must employ rather ad hoc assumptions about who is eating whom (which predator size class feeds on which prey size class).

In size-based models, trophic links of predators in a given size class are expressed by a feeding kernel, a generally unimodal function of prey size. Empirical studies on the kernel position seem to reflect allometries in the gathering and hunting abilities of consumers, but leave considerable scatter (Hansen et al. 1994). The patterning of these data indicates that the slope between logarithmic optimal prey size and predator size is $<1$; this conflicts with a simple linear relation as assumed by e.g. Armstrong (2003). In the most recent review study of Fuchs \& Franks (2010), the ratio of predator size to (ingested) prey size for distinct plankton grazer groups ranges on average over 1 to 2 orders of magnitude. Copepods, as the most prominent group, even display a ratio scatter over nearly 3 orders of magnitude. As a consequence of the poor precision of universal size relationships, the role of size as a trait has been criticized or replaced by different notions (DeMott 1989, Cózar et al. 2008, Pahlow \& Prowe 2010).

Feeding tactics or preferential behavior have been used in many descriptions and interpretations of plankton-plankton interactions (DeMott 1988, Verity 1991, Jeschke et al. 2004), but have not yet been incorporated into a more general and quantitative theory.

This study proposes that, at a given predator body size, feeding type and optimal prey size are hard- wired so that feeding type constitutes a second determinant of the feeding kernel position. With this new variable, food web models become more flexible to resolve changes in trophic interaction that have rarely been addressed to date. For example, various jellyfish, copepod, and microzooplankton species are able to switch between ambush and suspension feeding modes, so that different optimal prey sizes can be realized by the same organism (Landry 1981, Goldman \& Dennett 1990, Saiz \& Kiørbø 1995).

The theory is built on the mutual relation between feeding type, predator body size, and optimal prey size. It aims to meld size- and behavior-related factors into new mechanistic formulations. The derivation should first make use of biomechanical principles in order to reach a generic dependency of optimal prey size on predator size that is valid across the entire plankton realm. The 'null model' of a simple linear relation will thereby be evaluated critically (and adjusted, if necessary). Based on a more precise account of variation in the prey size ratio, the derivation will introduce a quantitative definition of feeding mode as a size-independent trait that codetermines optimal prey size.

A biomechanically-derived allometry in optimal prey size will start by considering how a specific morphotype can be re-scaled in size. Size increases in plankton organisms here are suggested to be delimited primarily by intra-body transport. This assumption refers to the hypothesis proposed by Wirtz (2011) that, beyond a threshold size, growth of autotrophic unicells is limited by intracellular resource gradients. Applying the concept to zooplankton, the necessity to transport fluids or chemical compounds throughout the body then re-shapes the body design. This is because a larger organism must develop a substantial fraction of structural biomass to sustain intrabody fluxes by which, for example, amino acids and monosaccharides are carried to functional end members. Evidence of size being relevant for intra-body transport can be found in the architecture of multicellular zooplankton. An interstitial and partly vascular system is common to crustaceans and many other zooplankton taxa. In calanoids, hemolymph circulation requires propulsion by a beating heart (Taylor 1982). Adult cnidarians possess a distributed gut structure for transport and absorption. The morehighly organized gastrovascular circulation system of scyphomedusae includes canals and branches of different size (Russell 1970), constituting an internal network topology, which complies with the hierarchical picture of intra-body transport put forward by West et al. (1999). A biomechanical account of intra- 
body transport will lay the basis for a functional formulation connecting predator body size, optimal prey size, and feeding type.

The proposed framework will be applied to existing review datasets of optimal prey sizes among all relevant planktonic taxa. Some implications for food web models will be outlined and more general conclusions drawn on the coevolution of predators and their prey.

\section{MODEL DERIVATION}

\section{Optimal prey size}

Plankton grazing is related to morphology, body size, or associated characteristics. To a large degree, the physical extents of a food item and its grazer determine the grazer's ability to reach and capture the prey item, to hold and handle it, to take it up (or suck it out), and to digest it smoothly. The range in which a food item matches a grazer's physical processing ability is known as the feeding size spectrum, and its center as the optimal prey size (Pastorok 1981, Hansen et al. 1994). The optimal prey size $D_{\text {opt }}$ reflects the shape and size of capture and digestive apparatuses (cilia, gut, or pallium). It is therefore specific to each predator organism and marks a key ecological trait. The trait variable $D_{\text {opt }}$ is used here in terms of the standard generalized size unit for the plankton, the equivalent spherical diameter (ESD).

The geometric dimensions of capture and digestive apparatus, however, do not completely explain variations in the size of the 'ideal' prey. These variations may occur for a single organism. A copepod can switch between an ambushing and a suspension feeding mode, which are both characterized by distinct values of $D_{\text {opt }}$ (Saiz \& Kiørbø 1995). Each $D_{\text {opt }}$ then corresponds to distinct morphological and behavioral features. Vice versa, differently sized consumers may have the same apparent $D_{\text {opt }}$ resulting from diverse feeding tactics. These tactics make variable use of feeding-related organs such as mandibles, meshes, or jaws and regulate the capture-related activities (swimming, feeding-current generation) to adjust the size of the targeted food particle.

\section{Feeding ratio}

In order to better distinguish between size- and non-size-related mechanisms that determine the optimal diet, it is instructive to define the feeding ratio $D_{\mathrm{opt}} / D_{\mathrm{Z}}$. The 1-dimensional extension of the feeding apparatus often correlates well with predator length or diameter $D_{\mathrm{Z}}$ (Burns 1968, Bogdan \& Gilbert 1984). As a consequence, the ratio $D_{\text {opt }} / D_{\mathrm{Z}}$ provides a quantitative and, in first order, size-independent measure of the feeding type because it cancels out a linear relation between $D_{\text {opt }}$ and $D_{\mathrm{Z}}$. At a specific $D_{\mathrm{Z}}$ a raptor will prefer larger prey (high $D_{\text {opt }} / D_{\mathrm{Z}}$ ) than a suspension feeder (low $D_{\text {opt }} / D_{\mathrm{z}}$ ). Handling of individual particles by the raptorial consumer yields a higher energetic return if the particle is large, whereas the filter feeder should target smaller items to avoid the risk of damaging the filter apparatus. An extreme case is a feeding size ratio that exceeds 1 ; a number of dinoflagellate species prey on cells larger than themselves. The ratio prescribes the same value for differently sized predators with equal body architecture, including the shape of the feeding apparatus, and equivalent feeding behavior; a doubling in $D_{\mathrm{Z}}$ then translates into a doubling in $D_{\text {opt }}$ (Fig. 1).

Preserving $D_{\text {opt }} / D_{\mathrm{Z}}$ under morphological scaling, however, implies independence of body architecture from absolute physical dimension. Morphometric relations (e.g. between mouth or corona width and body volume) realized in a given size class would have to be feasible in other size classes as well. This

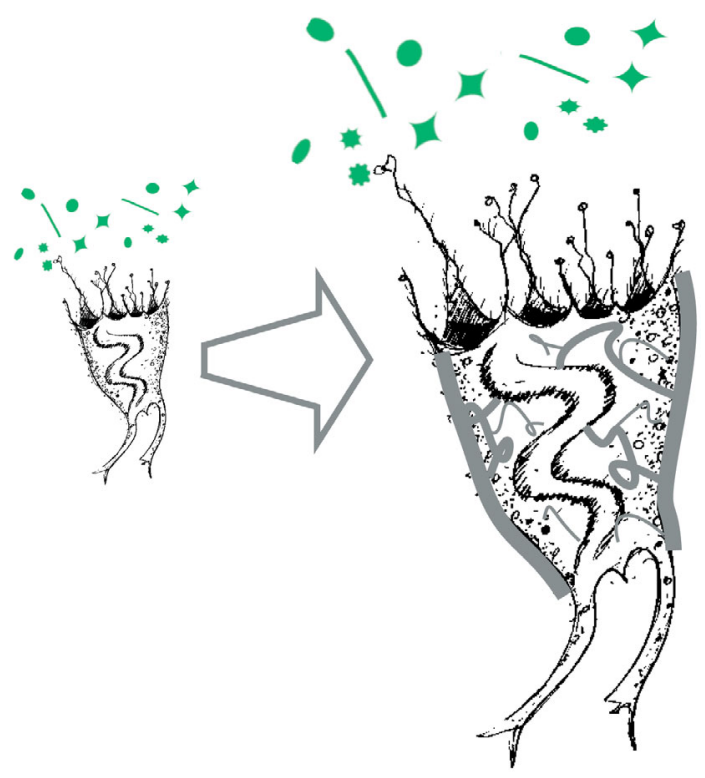

Fig. 1. Evolutionary up-scaling of a small predator (left, with ancestral equivalent spherical diameter, ESD, $D_{\mathrm{Z}}^{0}$ ) to a larger one (here, $D_{\mathrm{Z}}=2 D_{\mathrm{Z}}^{0}$ ). The doubling in body diameter necessitates additional transport-related structures (sketched as gray veins, corresponding to $D_{Z \text {,transp.). While leaving }}$ the form of the feeding-related apparatus unchanged, new structural elements increase the total volume. This additional dilation describes a non-isometric up-scaling 
may be too strong an assumption, as there are no identical planktonic morphotypes crossing many orders of magnitude in ESD.

\section{Non-isometric scaling of optimum prey size}

The tendency of growing structural complexity from small protists to larger aquatic invertebrates corroborates the importance of internal transport and supportive active components. If the amount of transport-related structural elements increases in larger organisms, geometric similarity cannot be maintained across size classes. As a result, morphology has to scale in a non-isometric way. If we consider, on an evolutionary time scale, the body enlargement of a species from a small, ancestral size $D_{\mathrm{Z}}^{0}$ to $D_{\mathrm{Z}}$ additional supportive elements require increasing body space. This additional demand modifies morphological proportions. Let $D_{\mathrm{Z} \text {,isomorph }}$ denote the isomorphic diameter $\left(=\mathrm{n} D_{\mathrm{Z}}{ }^{0}\right.$, with an arbitrary scaling factor $\left.\mathrm{n}\right)$ and $D_{Z \text {,transp }}$ the incremental 'deformation':

$D_{\mathrm{Z}}=D_{\mathrm{Z}, \text { isomorph }}+D_{\mathrm{Z} \text {,transp }}=\mathrm{n} \times\left(1+\Delta_{\text {transp }}\right) \times D_{\mathrm{Z}}^{0}$

$\Delta_{\text {transp }}$ measures the non-isomorphic body extension relative to $D_{Z, \text { isomorph }}$.

According to the metabolic theory of ecology, the number of additional structural elements scales with body volume by the power exponent $\varepsilon$ (West et al. 1999, Brown et al. 2004). In order to transfer the scaling relation into our ESD-based notation, it must be formulated from and into 1 dimension. This requires 2 consecutive divisions of the exponent by 3 . The first division converts the scaling unit volume to ESD, the second transforms a volumetric number into a 1dimensional dilation. In total, this leads to an effective diameter exponent of $\varepsilon / 9$. The size-dependency of the dilation introduces a scaling law into the relative body enlargement $\Delta_{\text {transp }}$

$$
\Delta_{\text {transp }}=\gamma^{\prime} \times\left(\left(\frac{D_{\mathrm{Z}}}{D_{\mathrm{Z}}^{0}}\right)^{\varepsilon / 9}-1\right)
$$

where the coefficient $\gamma^{\prime}$ transforms a (relative) increase in structural volume for intra-body transport (vessels, pumping organs) into (relative) ESD units. In principle, the factor $\gamma^{\prime}$ can be calculated based on the vessel volume of the 'prime' isomorph, but is treated here as a universal parameter. Subtraction by 1 in Eq. (2) ensures that the smallest (ancestral) isomorph (with size $D_{\mathrm{Z}}{ }^{0}$ ) has no additional need of transport-related biovolume.

Recalling the interpretation of the feeding size ratio, the parameter $r$,

$$
r=\frac{D_{\text {opt }}}{D_{\mathrm{Z}, \text { isomorph }}}
$$

represents the predation mode in the absence of deformation. Only the body part measured by $D_{\mathrm{Z} \text {,isomorph }}$ is isometrically connected to the feeding apparatus.

The deformation $\Delta_{\text {transp }}$ of the original architecture makes a small but important difference during evolutionary arms races (Smetacek 2001). As the prey species become slightly bigger, this triggers an adaptive increase in the optimal prey size by $\delta D_{\text {opt }}$. If the coevolving predator species maintains its feeding mode characterized by $r$ (Eq. 3), it undergoes a marginal size change by $\delta D_{\mathrm{Z}}$ that exceeds an isometric upscaling by $\delta D_{\mathrm{Z} \text {,isomorph. }}$. Such a total increment $\delta D_{\mathrm{Z}}$ has to account for non-scalable transport components given by the relative ESD enlargement $\delta D_{Z \text {,transp }}$ $\left(=\Delta_{\text {transp }} \times \delta D_{\mathrm{Z}}\right)$. For the marginal increment in the feeding apparatus $\delta D_{\mathrm{Z} \text {,isomorph }}$, and the corresponding marginal changes in optimal prey size and consumer size, this means

$$
\begin{gathered}
\delta D_{\mathrm{Z}, \text { isomorph }}=\delta D_{\mathrm{Z}}-\delta D_{\mathrm{Z}, \text { transp }} \\
\delta D_{\text {opt }}=r \times\left(\delta D_{\mathrm{Z}}-\delta D_{\mathrm{Z} \text {,transp }}\right) \\
\quad=r \times\left(1-\Delta_{\text {transp }}\right) \times \delta D_{\mathrm{Z}}
\end{gathered}
$$

For decreasing increments, Eq. (6) converges to a differential equation which, however, cannot be solved analytically because of the non-linear dependency of $\Delta_{\text {transp }}$ on predator size $D_{\mathrm{Z}}$ in Eq. (2). An approximate solution is therefore derived in Appendix 1:

$$
D_{\text {opt }}=r D_{\mathrm{Z}} \mathrm{e}^{-\gamma \ell_{\mathrm{Z}}^{2}} \quad \text { with } \ell_{\mathrm{Z}}=\log \left(D_{\mathrm{Z}} / D_{0}\right)
$$

In this work, the basis of the logarithmic function is e, thus $\log (\mathrm{x})=\ln (\mathrm{x})=\log \mathrm{e}(\mathrm{x})$. Optimal prey size increases with growing predator size, but with a decreasing slope. This decline in the slope is parameterized by the coefficient $\gamma$ (proportional to the specific structural volume increase $\gamma^{\prime}$, see Appendix 1). Given the quadratic dependency on $\log \left(D_{\mathrm{Z}} / D_{0}\right)$, the deviation from a linear relationship is most apparent in macroscopically large plankton taxa.

\section{Feeding type as quantitative trait}

The functional relationship between optimal prey size and predator size derived above introduces the scale-independent feeding size ratio $r$. This ratio takes a specific value for each consumer in a given feeding mode. In order to reduce the variability in $r$ due to the high diversity in feeding modes, the ratio 
is averaged over the entire plankton domain, using available empirical value pairs $D_{\mathrm{Z}}$ and $D_{\text {opt }}$ (see next subsection).

$$
\overline{D_{\text {opt }}}=\bar{r} D_{\mathrm{Z}} \mathrm{e}^{-\gamma \ell_{\mathrm{Z}}^{2}}
$$

This average ratio $\bar{r}$ accounts for the suggested effect that a large planktonic consumer species has more structural features not dedicated to feeding than a smaller one. A large consumer will therefore deal with proportionally smaller prey than a small-sized related species using the same feeding strategy.

The reference grazing ratio $\bar{r}$ characterizes a prototype or 'neutral' planktonic feeding strategy which is both an ambushing and a filtering type (like a fisherman targeting a small swarm using a net). Applied to a single predator size class, the neutral value $\bar{r}$ defines the feeding size ratio that distinguishes between ambushing and filtering aspects in the make-up of the body architecture and organ toolset or in the behavioral repertoire. The relation to this neutral value describes the 'activity' of specific tactics, compared to the neutral mode. This relational activity describes swimming velocity (e.g. relative to the average in the size class), directionality of swimming, up- and down-regulation of capturing activity (e.g. of a feeding current), usage of weapon tools or toxins, or the number of items processed in parallel.

A mechanistic interpretation of the feeding ratio that is based on the relation to the average value $r$ becomes facilitated by a mathematical transformation: the logarithm of a (re-normalized) ratio defines the feeding mode variable $m$ :

$$
\begin{aligned}
m & =\log (r / \bar{r}) \\
& =\log \left(D_{\text {opt }} / \overline{D_{\text {opt }}}\right) \\
& = \begin{cases}<0: & \text { passive, i.e. filtering } \\
>0: & \text { active, i.e. ambushing }\end{cases}
\end{aligned}
$$

where $m$ quantifies the relative activity of the feeding mode without any allometric effect. Negative values express a 'passive' strategy (no or slow swimming, unspecific prey targeting). As a variable defined in relation to an average mode, 'passiveness' lacks an imprinted lower limit. In principle, $m$ can be a very large negative number. Also within the coarse classification of suspension feeding, there might be differences with respect to aspects such as feedingcurrent regulation. The quantification works similarly in the other direction: positive values characterize active, directional chasing, and $m=0$ is attributed to a neutral or mixed strategy.

By construction, the normalized feeding type $\mathrm{m}$ follows from a known value of $D_{\text {opt }}$. It can, in turn, be used as an independent trait variable that determines the optimal prey size through Eqs. (7)-(9). Feeding type $m$ then forms a quantitative ecological trait that together with body size controls trophic relationships in plankton food webs.

\section{Data compilation and parameter fit}

Empirical estimates of optimal prey size $D_{\text {opt }}$ in the plankton are to date best documented within the extensive compilation of Hansen et al. (1994). To these data, the values given in the Appendix of Fuchs \& Franks (2010) were added. This second review dataset covers larger taxa, especially gelatinous plankton for which the majority of values derive from Purcell (1981a). The minimal-maximal range values reported by Fuchs \& Franks (2010) were transformed to $D_{\text {opt }}$ by taking the average of their logarithm. In most cases, this procedure resulted in values that were close to the optimal prey size estimates of Hansen et al. (1994), as cross-checked using the overlapping entries of both datasets. These overlapping values were removed subsequently. The 2 compilations were extended by available literature values for various copepod species from Landry \& Fagerness (1988), Euchaeta elongata from Greene \& Landry (1985), Brachionus plicatilis from Hansen \& Hansen (1997), Aurelia aurita and Cyanea capillata from Martinussen \& Båmstedt (1999), Stoecker et al. (1987), Ishii \& Tanaka (2001), Olesen (1995), and Barz \& Hirche (2005), and Doliolum denticulatum and Penilia avirostris from Katechakis et al. (2004). $D_{\text {opt }}$ for Gyrodinium spirale was shifted from $32 \mu \mathrm{m}$ (Hansen et al. 1994) to $23 \mu \mathrm{m}$ in order to render the data shown by Hansen (1992) consistent with a log-normal feeding kernel. This points to the uncertainty in estimates of $D_{\text {opt }}$ with respect to the number of feeding experiments, since the 'real' optimal prey may not be contained in the experimental series underlying the evaluation of $D_{\text {opt }}$.

The integrated dataset contains 159 individual estimates of optimal prey sizes. It enables an evaluation of the average feeding ratio $\bar{r}$ that should represent all planktonic grazers. Minimization of the deviation between empirical $D_{\text {opt }}$ and $D_{\text {opt }}$ calculated from Eq. (7) (using fminsearch in MATLAB) gave $\bar{r}=0.16$ and $\gamma=0.02$. Subsequent fits of $r$ (by application of Eq. 7) to data of individual taxa were normalized to obtain the taxon-specific relative feeding mode $m$ via Eq. (9). In addition, a linear regression without intercept was performed on $D_{\mathrm{Z}}$ and $D_{\text {opt }}$ using the taxonspecific data. 


\section{RESULTS AND DISCUSSION}

\section{Taxonomic differences in non-isometric scaling and feeding type}

Trophic relationships in the entire plankton domain are displayed separately for individual taxonomic groups in Fig. 2. In each group, optimal prey size increases with consumer size. Scattering of $D_{\text {opt }}$ around the linear regression lines turns out to be moderate; it becomes more substantial only for larvae of invertebrates and jellyfish (ctenophores, scyphomedusae). Surprisingly few singular values point to possible outliers, such as 1 extreme $D_{\text {opt }}$ reported for the siphonophore Rhyzophysa eysenhardti feeding exclusively on fish larvae (Purcell 1981a,b). Part of the variability in observed estimates of $D_{\text {opt }}$ can be explained by differences in experimental design. These specifics comprise culture conditions (e.g. physiological pre-conditioning or different water temperatures), non size-related features of prey organisms that may be distinguished by chemical composition or shape, or the number of underlying feeding experiments (see correction of the value
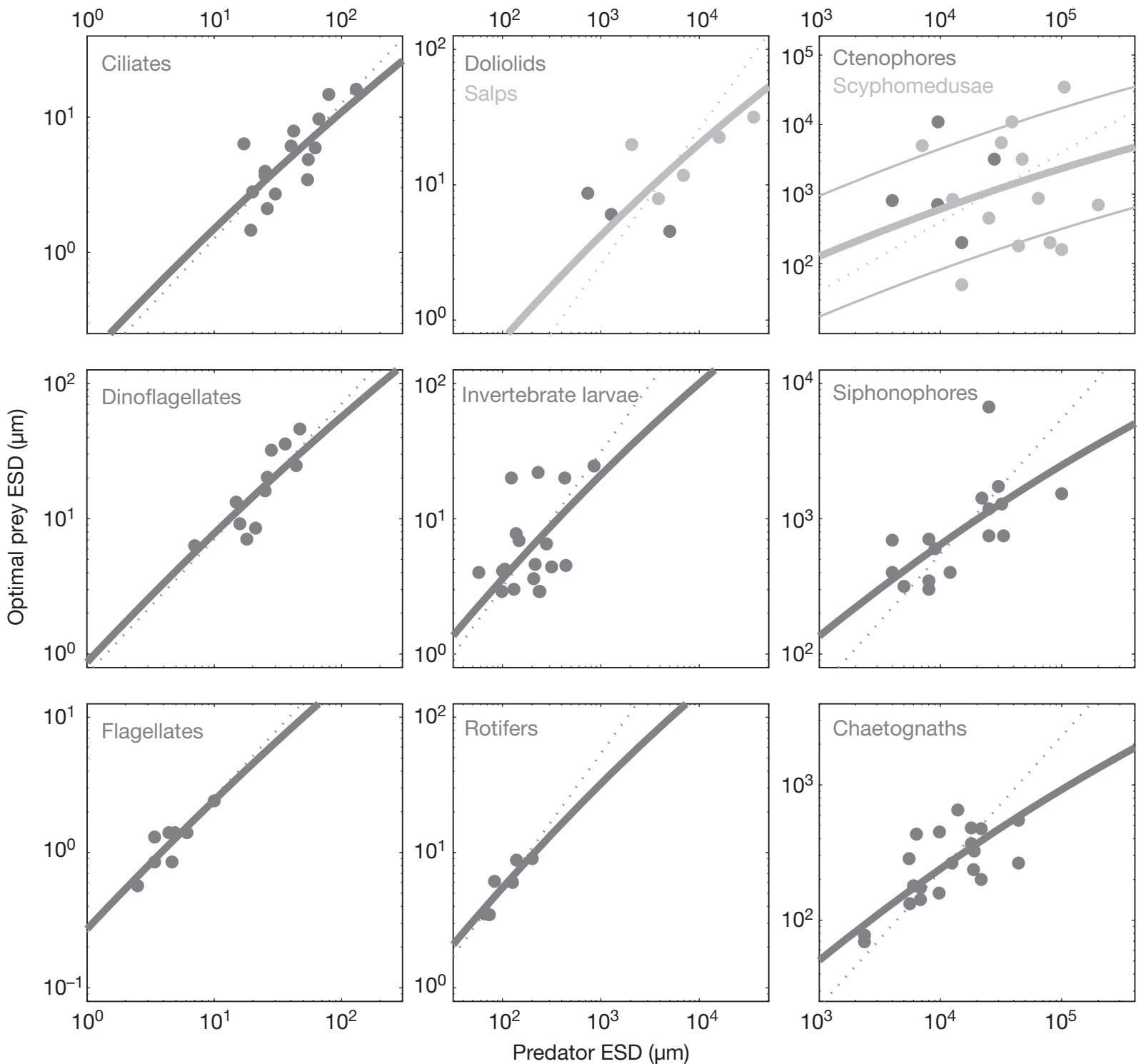

Fig. 2. Optimal prey size versus predator body size for diverse taxonomic groups. Experimental data, mostly compiled by Hansen et al. (1994) and Fuchs \& Franks (2010), are compared on logarithmic scales to 2 functions: the non-isometric scaling Eq. (7) (thick continuous line) and a linear regression without intercept (dotted line). In the panel for ctenophores, the nonisometric function is replicated with logarithmic offsets \pm 2 (thin continuous lines). Calibrated values of the feeding mode $r$ (or $m$ ) and regression coefficients are listed in Table 1. ESD: equivalent spherical diameter 
for Gyrodinium spirale documented above). For doliolids and ctenophores, the amount of data is too small to allow statistically meaningful analysis. These data were therefore merged into those of related taxonomic groups.

Despite methodological uncertainties inherent to the variability in the data, the linear regression as well as the non-isometric scaling model Eq. (7) provide relatively accurate fits. Residual errors are small in most cases, especially in microzooplankton (Table 1). In the macroscopic size range, by contrast, the non-isometric model is more compliant with the data than a linear model.

For chaetognaths, siphonophores, and salps, root mean square deviations of the non-isometric model are reduced by about $25 \%$ compared to the linear regression. This reduction is not negligible in units of log-ESD. For example, salps can play a major role in pelagic food web dynamics (Fortier et al. 1994). An enhanced description of their size-based trophic linkages may thus be beneficial for ecological modeling.

\section{Distinct feeding modes}

The accuracy of the non-isometric scaling model after calibration of the feeding mode $m$ for individual taxonomic groups indicates that feeding mode as a trait is rather group specific, with the (possible) exception of ctenophores and scyphomedusae, and copepods as a whole. Fitted values of $m$ listed in Table 1 (evaluated using the mean feeding ratio $\bar{r}=0.16$ ) start from negative numbers represent-

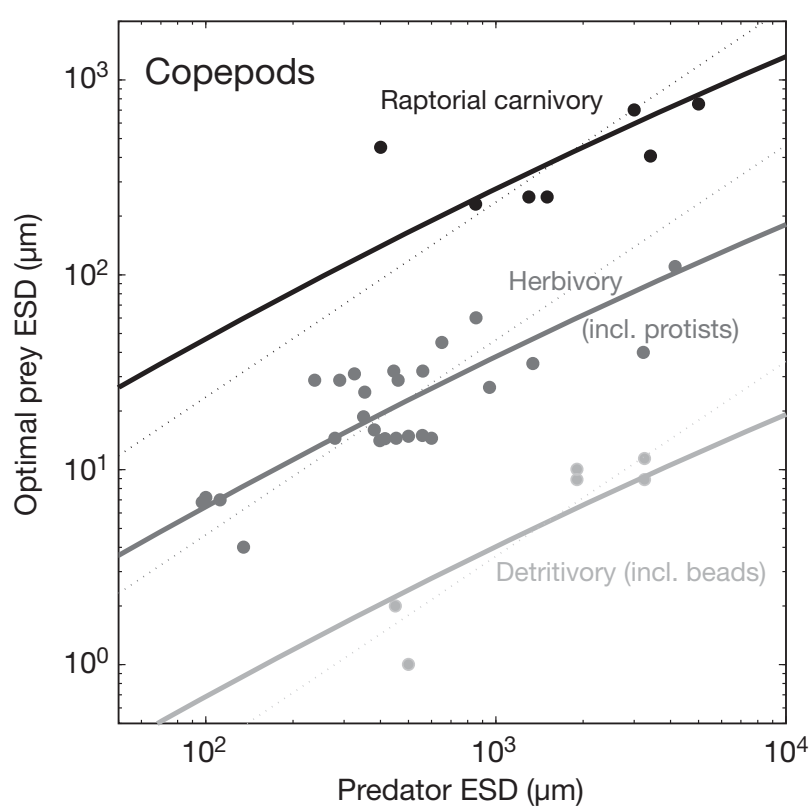

Fig. 3. Optimal prey size versus predator body size plotted for copepods only, see legend of Fig. 2 for definition of the thick continuous and the dotted lines. ESD: equivalent spherical diameter

ing passive suspension feeding (e.g. $m \simeq-1$ for larval invertebrates). Positive numbers indicate active ambushing, which includes, for example, usage of shortdistance weapons or piercing of cell walls. These mode values are found for various gelatinous predators (0.7-0.8) and for dinoflagellates (1.7).

Feeding size ratios of copepods reveal 3 separate clusters (Fig. 3). Each cluster represents a distinct major feeding mode. The 3 modes are characterized

Table 1. Calibration results of linear regression slopes (left) and of the non-isometric scaling model (right; fitted value of the feeding mode $m=\log (r / \bar{r})$, Eq. 7). Differences between predicted and reported value for optimal prey size are given as root mean squares (RMS). For each taxonomic group, the number of data pairs $(N)$ is added, and the a priori known major feeding type displayed (A: active, P: passive, M: mixed)

\begin{tabular}{|c|c|c|c|c|c|c|}
\hline \multirow[b]{2}{*}{ Group/mode } & \multirow[b]{2}{*}{ Tyрe } & \multirow[b]{2}{*}{$N$} & \multicolumn{2}{|c|}{ Linear regression } & \multicolumn{2}{|c|}{ Non-isometric scaling } \\
\hline & & & Slope & RMS & Feeding mode $m$ & RMS \\
\hline Flagellates & M & 8 & 0.258 & 0.09 & 0.53 & 0.09 \\
\hline Dinoflagellates & A & 11 & 0.710 & 0.15 & 1.69 & 0.16 \\
\hline Ciliates & M & 15 & 0.127 & 0.19 & 0.04 & 0.19 \\
\hline Rotifers & $\mathrm{P}$ & 6 & 0.054 & 0.08 & -0.65 & 0.07 \\
\hline Invertebrate larvae & $\mathrm{P}$ & 18 & 0.031 & 0.32 & -1.07 & 0.30 \\
\hline Chaetognaths & M & 20 & 0.023 & 0.25 & -0.20 & 0.19 \\
\hline Siphonophores & $\mathrm{A}$ & 16 & 0.055 & 0.30 & 0.79 & 0.26 \\
\hline Doliolids \& salps & $\mathrm{P}$ & 8 & 0.003 & 0.41 & -2.68 & 0.28 \\
\hline Ctenophores \& scyphomedusae & M & 18 & 0.040 & 0.87 & 0.73 & 0.80 \\
\hline \multicolumn{7}{|l|}{ Copepods } \\
\hline Detritivory & $\mathrm{P}$ & 6 & 0.004 & 0.15 & -2.73 & 0.19 \\
\hline Herbivory & M & 26 & 0.046 & 0.23 & -0.48 & 0.19 \\
\hline Carnivory & A & 7 & 0.236 & 0.30 & 1.50 & 0.22 \\
\hline
\end{tabular}


by a roughly equidistant sequence of $m(-2.7,-0.5$, 1.5). The data for herbivorous copepods approach either of the 2 fitting lines for this cluster only occasionally, suggesting a further division into 2 submodes. Similarly, intermediate $D_{\text {opt }}$ values are not observed between the 3 major clusters. Feeding type hence seems to define a discontinuous and disjunctive feature of (crustacean) predators. The maintenance of distinct modes can be interpreted as an economic adaptation at the taxonomic level to a wide prey size spectrum; by employing only a small number of diverse, feeding-related organs and a limited behavioral repertoire, large copepods are able to shift $D_{\text {opt }}$ and $m$ significantly. This switch can be realized by 1 species or by a re-organization of the copepod assemblage. Through a switch from detritivory to carnivory operated in the same size class, optimal prey ESD can be increased by more than 2 orders of magnitude, equivalent to about 7 orders of magnitude in volume. Most organisms in this range are potential prey since $D_{\text {opt }}$ denotes the center of the feeding kernel, which is, in turn, wider than 1 (in units of log-ESD, K. Wirtz unpubl.). A distance of 2 in the $m$-sequence thus describes a complementary and effective kernel combination by crustaceans. At a given predator size, crustaceans can cover a prey spectrum that spans-if we add a typical kernel width - a log-diameter factor of $4+2$ (corresponding to nearly 8 orders of magnitude in volume). Extreme broadness in the prey spectrum and flexibility in the feeding kernel position may be two of the reasons for the predominant role of crustaceans in many aquatic, particularly marine, food webs.

An extensive diet breadth is also known for gelatinous plankton. For example, medusae possess an array of passive and active grazing strategies. With the latter they may capture bigger prey such as other medusae or fish. For this, medusae and other gelatinous groups such as siphonophores carry powerful biochemical and micro-physical tool sets (e.g. toxin production in cnidocysts, ultrafast expulsion of venoms). In their suspension-feeding mode, many gelatinous species maintain semi-active control of the prey spectrum to be ingested. The postcontact capture success of the ctenophore Pleurobrachia is, for example, correlated to the swimming speed of crustacean prey (Greene et al. 1986), since these may detect and escape the feeding current. Feeding-current speed, in turn, can be subject to adaptive modifications. By actively down-regulating the speed, ctenophores and scyphomedusae may change the position of the prey size kernel towards bigger and faster organisms ('stealth predation'): below a threshold in shear stress critical for detection, various larger crustaceans stay in the feeding current, although their swimming speed would allow an escape (Raskoff 2002). This example for the behavioral diversity in medusae (e.g. Colin et al. 2006) means a total range of 4 in the new feeding mode variable $m$. The same value was already identified for copepods (cf. grey lines in topright diagram of Fig. 2), and, as for copepods, may explain why gelatinous zooplankton is prominently distributed at a global scale.

Ciliates, in comparison, seem to lack distinct or significantly variable grazing types. Although a diversity in feeding strategies has been attributed to ciliates by e.g. Kivi \& Setälä (1995) or Tirok \& Gaedke (2007), species-specific optimal prey sizes only moderately deviate from the average curves (Fig. 2). Feeding size ratios for this microzooplankton group are more centered around the regression line than for medusae or crustaceans, which could be an artifact of the limited amount of data (see larger ciliates species above a cell volume of $5 \times$ $10^{4} \mathrm{~m}^{3}$ ). Ciliates, interestingly, have a mean mode value $m$ close to 0 (resulting from $r \approx \bar{r}$ in Eq. 8). In this respect, they can be regarded as 'prototype' plankton grazers because they constitute the mode separation line in their size class, whereas all other plankton groups presented in the data compilation either exhibit a clearly active $(m>0.5)$ or passive $(m<-0.5)$ mode. Only chaetognaths, a rather rare group in many habitats, also reveal a neutral strategy $(m=0.2)$.

\section{Quantification of feeding mode}

Over the entire plankton domain, the non-linear mode separation curve ( $m=0$ isoline in Fig. 4) describes the part of $D_{\text {opt }}$ that is determined by predator size alone. Remaining variability perpendicular to this line was tentatively classified here as 'activity'. According to this interpretation, the curve defines the first (non-linear) principal component of a coordinate system spanned by 2 ecological traits. Grazing activity $m$ constitutes the second principal trait axis; at a given predator size, $m$ describes whether a species moves through the fluid to capture a single item or moves the fluid itself to filter many items. Cruising as an active and feeding-current generation as a passive strategy have already been contrasted by Greene (1985) in his classification of zooplankton prey selection tactics. Activity as a relational notion for feeding type $m$ is to some extent linked to the swimming speed during grazing, relative to the sur- 


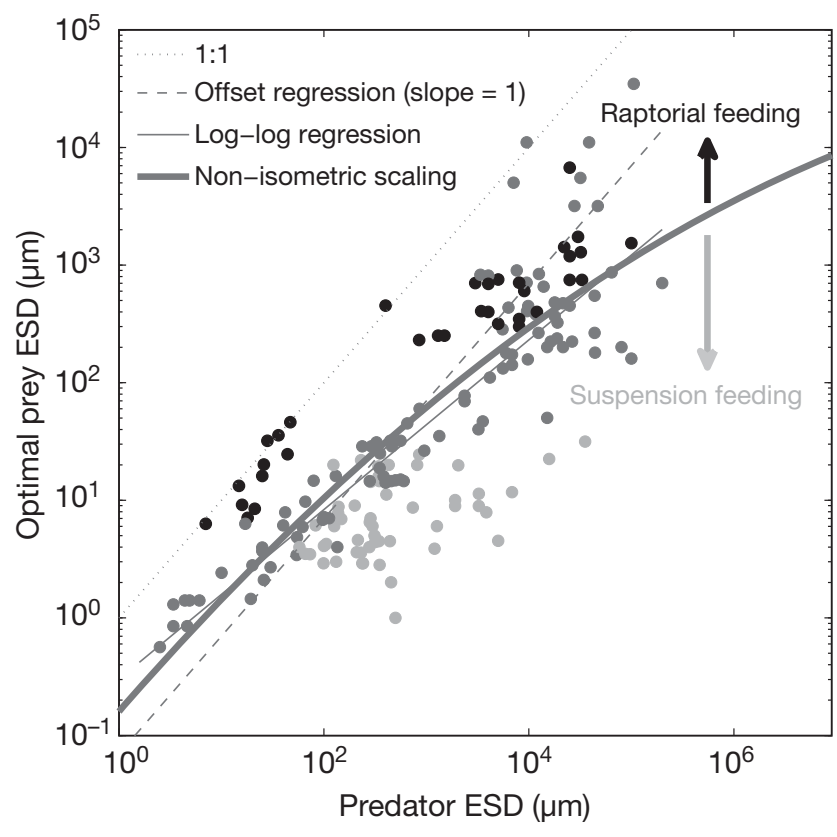

Fig. 4. Optimal prey size versus predator body size plotted for the entire zooplankton. Four statistical models are displayed for comparison: (1) the 1:1 line revealing that, apart from dinoflagellates in the 10 to $80 \mu \mathrm{m}$ range, few species in higher size classes chase prey of the same size, (2) a calibrated linear model for optimal prey size $D_{\mathrm{opt}}=0.07 D_{\mathrm{Z}}$ where $D_{\mathrm{Z}}$ is prey diameter, (3) a $\log$-log linear regression $\left(\log D_{\text {opt }}=-1.3+0.75 \log D_{\mathrm{Z}}\right)$, and (4) the non-isometric curve $(m=0$ isoline, Eq. 8). Feeding mode as a second trait axis is visualized by arrows. These either point to active strategies of raptors or passive strategies of suspension feeders. Their length represents changes in feeding type $m$ by 1 (upwardpointing arrow) or 2 (downward-pointing arrow). ESD: equivalent spherical diameter

rounding water and relative to the average in the size class. In the logic of the new 'trait coordinate' system, very negative values of $m(<-2)$ represent a deeply passive strategy where an existing water current replaces the generation of additional feeding flows (Jeschke et al. 2004). In the vertebrate domain, whales feasting on krill are best known for this strategy; their feeding size ratio corresponds to mode values $m \ll-5$. Compared to this example, the tactics of most filter feeders, with $m \sim-2$, still include some active aspects.

Feeding types of the studied taxonomic groups were classified beforehand into the categories of 'active', 'passive', or 'mixed' mode based on qualitatively known grazing characteristics (Table 1). In Fig. 4, all measured size ratios above the $m=0$ isoline belong to species of 'active' groups, while nearly all below the isoline belong to 'passive' species. Feeding mode hence seems to be a group-specific trait.

\section{Limited role of predator size}

The $m=0$ isoline curve derived from non-isometric scaling predicts the overall trend in $D_{\text {opt }}$ as a function of $D_{\mathrm{Z}}$ more precisely than a direct proportionality (with slope of 1 in the log-log diagram, Fig. 4). A linear relationship can be excluded by a $t$-test $(t=8.7$, $\mathrm{p}<0.0001)$. This result is important to note since linearity between $D_{\text {opt }}$ and $D_{\mathrm{Z}}$ has been assumed by some modeling studies (Armstrong 2003, Poulin \& Franks 2010). There is, however, no difference between the non-isometric curve and the log-log linear regression. Their equally high skill $\left(r^{2}=0.69, \mathrm{p}<\right.$ $0.0001)$ underlines the relevance of predator size as a predictor variable. The regression line (with slope 0.75 , equivalent to an absolute size scaling exponent of 3/4) may in some applications replace the slightly more complicated non-isometric function (quadratic term in Eq. 7). The exact slope of the log-log regression is, however, sensitive to the selection of measurements; the data compiled by Hansen et al. (1994) reveal a slope of 0.45 , as they do not contain the gelatinous plankton with their high values, in $D_{\text {opt }}$. Root mean square deviations in $\log \left(D_{\text {opt }}\right)$ of all 3 models (slope-one regression, log-log linear regression, and non-isometric function) vary around 0.6 and do not differ significantly, in contrast to their error distribution. Ideal food sizes of protistan zooplankton are systematically underestimated by the slope-one regression. In the macroscopic size range, the slope-one regression overestimates $D_{\text {opt }}$ of large and suspensionfeeding scyphomedusae by a factor of 40 . If we disregard the extreme datum with the largest $D_{\text {opt }}$ of the entire compilation, which represents Cyanea capillata feeding on similarly sized Aurelia aurita (Martinussen \& Båmstedt 1999), the non-isometric model does not deviate from empirical $D_{\text {opt }}$ estimates by more than a factor of 10 for the entire dataset. This feature of the non-isometric curve means that nearly every speciesspecific value of $D_{\text {opt }}$ can be reached, starting from the basal reference line, by assigning to $m$ an absolute value lower than 2 (range displayed by gray arrow in Fig. 4). The scatter, however, is not uniform. Small predators with ESD below $10^{2} \mu \mathrm{m}$ and larger ones above $10^{4} \mu \mathrm{m}$ tend to be raptorial $(m=0 \ldots 2)$. In the intermediate size range, by contrast, most species feed passively $(m=-2 \ldots 0)$. During the evolution of morphotypes and feeding strategies, trade-offs have probably interlinked the 2 traits body size and feeding mode.

Mechanical constraints on evolutionary size and feeding mode adaptation and the species diversity resulting from these constraints can be relevant agents for successional shifts in consumer communi- 
ties. Successional changes can be realized along either $D_{\mathrm{Z}}$ or $m$, but not always fully independently. Some combinations may also be excluded, such as $m$ $\geq 1$ in the $500 \mu \mathrm{m}$ class. In addition, succession in consumer communities has to acknowledge nonsize-related properties of the prey. A shift in optimal prey size has implications for dealing with altered prey motility, shape, or chemical composition/defense. For example, the ciliate community in Lake Constance displays alterations with respect to both feeding mode and mean body size (Figs. $4 \& 5$ in Tirok \& Gaedke 2007). Such shifts in $D_{\mathrm{Z}}$ and $m$ can be stimulated by compositional changes in prey abundance, size structure, and quality. This example highlights the explanatory power of the (quantitative) feeding mode as a major ecological trait, side by side with body size. Together, the 2 variables build a trait space where the co-existence of competing species is more likely to occur than in a 1-dimensional (size) picture. Non-allometric traits may therefore contribute to the generation and maintenance of the huge diversity found in plankton food webs.

\section{Consequences for food web structure}

This study provides some new insights into application limits of allometric relations in food web interactions. In 'allometric diet breadth models' (ADBMs), empirical size-scaling rules combined with optimal foraging theory are used to generate distributions of foraging traits such as handling time and resulting optimal body size ratio (Petchey et al. 2008, Thierry et al. 2011). Although ADBMs can explain a reasonable share of complex trophic links found in terrestrial, freshwater, and marine ecosystems, their skill can still be improved either by re-calibrating the underlying allometries (Williams et al. 2010) or by using simpler niche models (Allesina 2011).

A related branch of research seeks to reconstruct food web structure by elucidating the relevance of evolutionary history (phylogeny, Rossberg et al. 2006, Bersier \& Kehrli 2008). Like ADBMs, phylogenetic approaches have found a considerable degree of non-allometric determinants (termed 'latent traits') in predator-prey size relations. These latent traits partly correlate with the phylogeny of the species (Rohr et al. 2010, Naisbit et al. 2011).

Major results of the phylogenetic studies comply with our findings insofar as they stress the importance of addressing both size- and non-size-related determinants of kernel position and width. As discussed above, these 2 groups of determinants may not be fully independent. In order to maintain a small predator-prey size ratio or, equivalently, the ability to chase large prey organisms, non-isometric scaling (with a quadratic $\log -D_{\mathrm{Z}}$ reduction of $\log D_{\text {opt }}$ ) predicts an increasing activity (sensu raptorial-like strategies) in larger planktonic animals. This pattern is also found in fish (Pearre 1986).

The approach presented in this study could help to refine universal size scaling laws as suggested, for example, for ADBMs. It could also provide a framework for linking morphological features and behavioral repertoires of predators and prey to their phylogenetic history. Its major improvement thus goes beyond a better data fit. The approach applies the same biomechanical constraints to predator-prey size variations within and between taxonomic groups. In doing so, it predicts and mechanistically explains the declining slope between $D_{\text {opt }}$ and $D_{\mathrm{Z}}$ in large zooplankton.

\section{SUMMARY AND CONCLUSIONS}

The feeding kernel defines a central component for realistic plankton food web models. Its position in the prey size space is here proposed to be largely determined by the 2 quantitative predator traits body size and feeding mode. The dependency of optimal prey size on predator body size deviates from a linear relationship. In order to describe that larger consumers increasingly face intra-body transport limitations, the non-isometric scaling model includes a quadratic $\log$-ESD correction for optimal prey size $\left(\log D_{\text {opt }}=m\right.$ $+\log D_{\mathrm{Z}}-\gamma \log D_{\mathrm{Z}}{ }^{2}$ ). This correction can be approximated by a log-log linear relation with slope 0.75 . For individual taxonomic groups at the $\mu \mathrm{m}$ scale, the non-isometric model performs like log-log regressions, but it much better reproduces the data for plankton at the $\mathrm{mm}$ to $\mathrm{cm}$ scale.

Feeding mode $m$ as the second determinant of the kernel position roughly quantifies the activity related to grazing, in terms of, e.g. swimming velocity (related to the fluid and to the average of the same size class). Feeding mode differences within major taxa such as crustaceans are organized in discrete clusters. This discretization of $m$ can be interpreted as an efficient means by which grazers cover a large prey size spectrum with a minimum repertoire of morphological and behavioral features.

The new theory introduces non-allometric aspects of plankton-plankton interactions in a quantitative way. Non-size-related factors should be categorized similar to the size-related ones in order to reach a 
consistent multi-trait description of trophic relations in the plankton. In this study, non-size traits have been treated only on the side of the predator, but not of the prey. An important step would be to account for the nutritional quality as expressed by the elemental stoichiometry of the prey (Hessen 1992, Mitra \& Flynn 2006). A future, more complete definition of trophic plankton traits thus has to mechanistically describe how escape or defense behavior, toxicity, stoichiometry, and shape factors of the prey influence the topology of aquatic food webs.

Acknowledgements. I thank M. Schartau and J. Bruggeman for valuable comments on an early version of the draft. L. Winter improved the English. I. Liffler-Wirtz drew the planktonic consumer in Fig. 1 and R. Schüller provided photographs of Acartia tonsa in different prey fields. I was supported by the Helmholtz Society via the program PACES. Three anonymous reviewers are acknowledged for instructive comments.

\section{LITERATURE CITED}

- Allesina S (2011) Predicting trophic relations in ecological networks: a test of the allometric diet breadth model. J Theor Biol 279:161-168

> Andersen KH, Beyer JE (2006) Asymptotic size determines species abundance in the marine size spectrum. Am Nat 168:54-61

Armstrong RA (2003) A hybrid spectral representation of phytoplankton growth and zooplankton response: the 'control rod' model of plankton interaction. Deep-Sea Res II 50:2895-2916

Baird M (2010) Limits to prediction in a size-resolved pelagic ecosystem model. J Plankton Res 32:1131-1146

> Banas N (2011) Adding rich trophic interactions to a sizespectral plankton model: emergent diversity patterns and limits on predictability. Ecol Model 222:2663-2675

- Barz K, Hirche H (2005) Seasonal development of scyphozoan medusae and the predatory impact of Aurelia aurita on the zooplankton community in the Bornholm Basin (central Baltic Sea). Mar Biol 147:465-476

> Bersier L, Kehrli P (2008) The signature of phylogenetic constraints on food-web structure. Ecol Complex 5:132-139

Bogdan KG, Gilbert JJ (1984) Body size and food size in freshwater zooplankton. Proc Natl Acad Sci USA 81: 6427-6431

Brooks JL, Dodson SI (1965) Predation, body size, and composition of plankton. Science 150:28-35

Brown JH, Gillooly JF, Allen AP, Savage VM, West GB (2004) Toward a metabolic theory of ecology. Ecology 85: 1771-1789

Bruggeman J, Kooijman S (2007) A biodiversity-inspired approach to aquatic ecosystem modeling. Limnol Oceanogr 52:1533-1544

Burns C (1968) The relationship between body size of filterfeeding Cladocera and the maximum size of particle ingested. Limnol Oceanogr 13:675-678

Cohen J, Pimm S, Yodzis P, Saldaña J (1993) Body sizes of animal predators and animal prey in food webs. J Anim Ecol 62:67-78
Colin SP, Costello JH, Kordula H (2006) Upstream foraging by medusae. Mar Ecol Prog Ser 327:143-155

Cózar A, García CM, Gálvez JA, Echevarría F (2008) Structuring pelagic trophic networks from the biomass size spectra. Ecol Model 215:314-324

DeMott W (1988) Discrimination between algae and artificial particles by freshwater and marine copepods. Limnol Oceanogr 33:397-408

> DeMott W (1989) Optimal foraging theory as a predictor of chemically mediated food selection by suspensionfeeding copepods. Limnol Oceanogr 34:140-154

> Fortier L, Le Fèvre J, Legendre L (1994) Export of biogenic carbon to fish and to the deep ocean: the role of large planktonic microphages. J Plankton Res 16:809-839

> Fuchs HL, Franks PJS (2010) Plankton community properties determined by nutrients and size-selective feeding. Mar Ecol Prog Ser 413:1-15

Goldman JC, Dennett MR (1990) Dynamics of prey selection by an omnivorous flagellate. Mar Ecol Prog Ser 59: 183-194

Greene C (1985) Planktivore functional groups and patterns of prey selection in pelagic communities. J Plankton Res 7:35-40

> Greene CH, Landry MR (1985) Patterns of prey selection in the cruising calanoid predator Euchaeta elongata. Ecology 66:1408-1416

> Greene C, Landry M, Monger B (1986) Foraging behavior and prey selection by the ambush entangling predator Pleurobrachia bachei. Ecology 67:1493-1501

> Hansen PJ (1992) Prey size selection, feeding rates and growth dynamics of heterotrophic dinoflagellates with special emphasis on Gyrodinium spirale. Mar Biol 114: 327-334

Hansen J, Hansen BW (1997) Zooplankton grazing and growth: scaling within the $2-2,000-\mu \mathrm{m}$ body size range. Limnol Oceanogr 42:687-704

> Hansen B, Bjornsen PK, Hansen PJ (1994) The size ratio between planktonic predators and their prey. Limnol Oceanogr 39:395-403

> Hessen D (1992) Nutrient element limitation of zooplankton production. Am Nat 140:799-814

> Ishii H, Tanaka F (2001) Food and feeding of Aurelia aurita in Tokyo Bay with an analysis of stomach contents and a measurement of digestion times. Hydrobiologia 451: 311-320

Jeschke JM, Kopp M, Tollrian R (2004) Consumer-food systems: why type I functional responses are exclusive to filter feeders. Biol Rev Camb Philos Soc 79:337-349

Katechakis A, Stibor H, Sommer U, Hansen T (2004) Feeding selectivities and food niche separation of Acartia clausi, Penilia avirostris (Crustacea) and Doliolum denticulatum (Thaliacea) in Blanes Bay (Catalan Sea, NW Mediterranean). J Plankton Res 26:589-603

Kivi K, Setälä O (1995) Simultaneous measurement of food particle selection and clearance rates of planktonic oligotrich ciliates (Ciliophora: Oligotrichina). Mar Ecol Prog Ser 119:125-137

> Landry M (1981) Switching between herbivory and carnivory by the planktonic marine copepod Calanus pacificus. Mar Biol 65:77-82

Landry M, Fagerness V (1988) Behavioral and morphological influences on predatory interactions among marine copepods. Bull Mar Sci 43:509-529

Martinussen MB, Båmstedt U (1999) Nutritional ecology of gelatinous planktonic predators. Digestion rate in rela- 
tion to type and amount of prey. J Exp Mar Biol Ecol 232:61-84

Mitra A, Flynn K (2006) Accounting for variation in prey selectivity by zooplankton. Ecol Model 199:82-92

- Moloney CL, Field JG (1991) The size-based dynamics of plankton food webs. I. A simulation model of carbon and nitrogen flows. J Plankton Res 13:1003-1038

Naisbit R, Kehrli P, Rohr R, Bersier L (in press) (2011) Phylogenetic signal in predator-prey body-size relationships. Ecology 92:2183-2189

Olesen NJ (1995) Clearance potential of jellyfish Aurelia aurita, and predation impact on zooplankton in a shallow cove. Mar Ecol Prog Ser 124:63-72

Pahlow M, Prowe AEF (2010) Model of optimal current feeding in zooplankton. Mar Ecol Prog Ser 403:129-144

Pastorok RA (1981) Prey vulnerability and size selection by Chaoborus larvae. Ecology 62:1311-1324

Pearre S Jr (1986) Ratio-based trophic niche breadths of fish the Sheldon spectrum, and the size-efficiency hypothesis. Mar Ecol Prog Ser 27:299-314

Petchey OL, Beckerman AP, Riede JO, Warren PH (2008) Size, foraging, and food web structure. Proc Natl Acad Sci USA 105:4191-4196

Poulin FJ, Franks PJS (2010) Size-structured planktonic ecosystems: constraints, controls and assembly instructions. J Plankton Res 32:1121-1130

Purcell J (1981a) Dietary composition and diel feeding patterns of epipelagic siphonophores. Mar Biol 65:83-90

Purcell J (1981b) Feeding ecology of Rhyzophysa eysenhardti, a siphonophore predator of fish larvae. Limnol Oceanogr 26:424-432

Raskoff K (2002) Foraging, prey capture, and gut contents of the mesopelagic narcomedusa Solmissus spp. (Cnidaria: Hydrozoa). Mar Biol 141:1099-1107

Rohr RP, Scherer H, Kehrli P, Mazza C, Bersier LF (2010) Modeling food webs: exploring unexplained structure using latent traits. Am Nat 176:170-177

Rossberg AG, Matsuda H, Amemiya T, Itoh K (2006) Food webs: experts consuming families of experts. J Theor Biol 241:552-563
Russell F (1970) The medusae of the British Isles. Cambridge University Press, Cambridge

Saiz E, Kiørbø T (1995) Predatory and suspension feeding of the copepod Acartia tonsa in turbulent environments. Mar Ecol Prog Ser 122:147-158

Smetacek V (2001) A watery arms race. Nature 411:745

Stoecker D, Michaels A, Davis L (1987) Grazing by the jellyfish, Aurelia aurita, on microzooplankton. J Plankton Res 9:901-915

Taylor E (1982) Control and co-ordination of ventilation and circulation in crustaceans: responses to hypoxia and exercise. J Exp Mar Biol Ecol 100:289-319

Thierry A, Petchey O, Beckerman A, Warren P, Williams R (2011) The consequences of size dependent foraging for food web topology. Oikos 120:493-502

Tirok K, Gaedke U (2007) Regulation of planktonic ciliate dynamics and functional composition during spring in Lake Constance. Aquat Microb Ecol 49:87-100

Verity P (1991) Feeding in planktonic protozoans: evidence for non-random acquisition of prey. J Eukaryot Microbiol 38:69-76

West GB, Brown JH, Enquist BJ (1999) The fourth dimension of life: fractal geometry and allometric scaling of organisms. Science 284:1677-1679

Williams RJ, Anandanadesan A, Purves D (2010) The probabilistic niche model reveals the niche structure and role of body size in a complex food web. PLoS ONE 5:e12092

Wirtz KW (2011) Non-uniform scaling in phytoplankton growth rate due to intracellular light and $\mathrm{CO}_{2}$ decline. J Plankton Res 33:1325-1341

Woodward G, Hildrew A (2002) Body-size determinants of niche overlap and intraguild predation within a complex food web. J Anim Ecol 71:1063-1074

Woodward G, Speirs D, Hildrew A (2005) Quantification and resolution of a complex, size-structured food web. Adv Ecol Res 36:85-135

> Zhou M, Carlotti F, Zhu Y (2010) A size-spectrum zooplankton closure model for ecosystem modelling. J Plankton Res 32:1147-1165

Appendix 1. Non-isometric scaling: approximated differential equation

The relative deformation $\Delta_{\text {transp }}$ given in Eq. (2) is rewritten here using the auxiliary variable $\ell^{\prime}=\varepsilon / 9 \log \left(D_{\mathrm{Z}} / D_{0}\right)=\varepsilon \ell_{\mathrm{Z}} / 9$ (with $\ell_{\mathrm{Z}}=\log \left(D_{\mathrm{Z}} / D_{0}\right)$, cf. Eq. 7), and a second-order Taylor series expansion in $\ell^{\prime}$.

$$
\Delta_{\text {transp }} / \gamma^{\prime}=\mathrm{e}^{\ell^{\prime}}-1 \approx \ell^{\prime}+1 / 2 \ell^{\prime 2}
$$

'Backward' second-order expansion in the very small argument $\gamma^{\prime} \ell^{\prime}\left(\gamma^{\prime} \ell^{\prime} \ll 1\right)$ produces

$$
1-\Delta_{\text {transp }}=1-\gamma^{\prime} \ell^{\prime}-\gamma^{\prime} / 2 \ell^{\prime 2} \approx\left(1-\gamma^{\prime} \ell^{\prime}\right) \times \mathrm{e}^{-\gamma / 2 \ell^{\prime 2}}
$$

with $\gamma=\gamma^{\prime} \varepsilon / 3$. The differential equation for the predator size, now formulated for the logarithmic size $\ell_{\mathrm{Z}}$, has an analytical solution (see Eq. 7):

$$
\frac{\mathrm{d} D_{\text {opt }}}{\mathrm{d} \ell_{\mathrm{Z}}}=r \times\left(1-\gamma \ell_{\mathrm{Z}}\right) \times \mathrm{e}^{\ell_{\mathrm{Z}}-\gamma / 2 \ell_{\mathrm{Z}}^{2}}
$$

Editorial responsibility: Alejandro Gallego, Aberdeen, UK
Submitted: July 15, 2011; Accepted: November 14, 2011

Proofs received from author(s): January 3, 2012 